\title{
Midiendo el capital social: una aproximación desde la participación ciudadana en gobiernos locales
}

\author{
Measuring social capital: An approach based \\ on citizen participation in local governments
}

\author{
Y. LORENA VÁSQUEZ-GONZÁLEZ \\ Universidad de Bogotá Jorge Tadeo Lozano \\ Como citar/Citation \\ Vásquez-González, Y. L. (2018). Midiendo el capital social: una aproximación desde la participación ciudadana \\ en gobiernos locales. Revista Española de Ciencia Política, 48, 103-128. Doi:https://doi.org/10.21308/recp.48.04
}

\section{Resumen}

Desde la década de 1990, el capital social se ha vuelto una variable frecuente en diversos campos de investigación de las ciencias sociales. En general, las contribuciones teóricas han destacado que este recurso se compone de dimensiones tales como redes, confianza y normas sociales. En la literatura sobre participación local, el capital social ha sido usualmente operacionalizado a partir de la observación de asociaciones civiles, enfocándose en las redes, pero dejando de lado el estudio sobre la confianza y las normas sociales. Con el ánimo de corregir esta brecha, el presente artículo trata de aportar nuevas herramientas de operacionalización que, basadas en campos como los community studies, el urbanismo o la teoría de redes, enriquezcan la capacidad analítica de esta variable. Reconociendo que el capital social es una variable que depende del contexto en el cual se enmarca y que, por ello, es inadecuado proponer una única manera de medirlo, este artículo reflexiona sobre las diferentes posibilidades metodológicas de abordar el capital social; en particular, sobre cómo operacionalizar una variable clave, pero a la vez ambigua en los estudios sobre participación local.

Palabras clave: participación ciudadana, capital social, gobiernos locales, operacionalización.

\begin{abstract}
Since the 1990s, social capital has become a frequent variable in various fields of social science research. In general, the theoretical contributions have highlighted that this resource is composed of dimensions such as networks, trust and social norms. In the literature on local participation, social capital has usually been operationalized based on the observation of civil associations, with a focus on the networks, but leaving the study of trust and social norms
\end{abstract}


aside. This article aims to fill this gap by seeking to provide new operationalization tools that, based on fields such as community studies, urban studies or network theory, will enrich the analytical capacity of the social capital variable. Recognizing that social capital is a variable that depends on the context that surrounds it and it is thus inappropriate to propose a single way of measuring it, this article reflects on different methodological possibilities of addressing it; particularly on how to operationalize a variable which is both fundamental and ambiguous in the studies on local participation.

Keywords: citizen participation, social capital, local government, operationalization.

\section{INTRODUCCIÓN}

Cada vez es más frecuente encontrar el término de capital social en estudios sobre áreas tan distintas como el desarrollo económico, la agricultura, la salud, la pobreza, el fortalecimiento democrático y, como se desarrollará en este artículo, la participación ciudadana en gobiernos locales. El origen del gran atractivo del capital social está en su pretendida capacidad de explicar macrofenómenos sociales a partir de la comprensión de dinámicas micro. Los estudios sobre este tema que parten del análisis de comportamientos individuales, buscan identificar relaciones causales que expliquen cuestiones tales como la democracia, el desarrollo económico y la cohesión social, entre otros. Sin embargo, la evolución del capital social como categoría analítica y explicativa de múltiples fenómenos sociales no ha estado exenta de polémica.

Los debates sobre este asunto se pueden agrupar en tres aspectos. El primero es el relativo a su conceptualización. Esto es central, ya que refiere a las características de este fenómeno y a su capacidad analítica respecto a diferentes problemáticas. La segunda fuente de críticas tiene que ver con la manera en la que operan los mecanismos que vinculan lo micro y lo macro, es decir, la identificación de las relaciones causales, en donde el capital social se ubica a menudo como variable explicativa de diferentes cuestiones. Y la tercera, refiere a la capacidad de medir y de demostrar dichas relaciones, que alude a la operacionalización del concepto, cómo convertirlo de un concepto teórico a uno empírico. En general, las investigaciones que hacen uso de la variable capital social se tienen que enfrentar a estos tres aspectos problemáticos.

Vinculado al análisis de las relaciones causales, la comprensión del capital social en su aspecto colectivo es lo que a juicio de Ostrom y Ahn (2003: 167) ha permitido el salto a un concepto más amplio, a la vez que complejo. Esta nueva perspectiva considera que el capital social comprende tanto aspectos estructurales (tales como conexiones entre personas y redes) como culturales (obligaciones, normas sociales, valores, confianza interpersonal, entre otros) (Van Deth, 2008: 151). En consecuencia, se señala que el capital social está compuesto por redes, confianza y normas sociales.

Una de las áreas en donde de manera creciente se ha hecho uso del concepto de capital social es en los estudios sobre participación ciudadana. La participación ciudadana directa en gobiernos locales ha tenido múltiples acepciones, y es un reflejo de la 
pluralidad de prácticas participativas alrededor del mundo. A pesar de esta diversidad, en general este concepto hace referencia a las diferentes actividades y prácticas que tienen como propósito que la ciudadanía influya directamente en la toma decisiones de los gobiernos locales sobre temas que les atañen. Estos procesos cuentan con algún tipo de reconocimiento del gobierno local aunque no fuera este quien impulsara y organizara la iniciativa (Lowndes y Pratchett, 2009; Avrtizer, 2010; Font, 2011). Los mecanismos participativos institucionalizados son creados por los gobiernos locales, bajo algún grado de legitimidad, con la finalidad de permitir cierta intervención de los ciudadanos comunes en el proceso de políticas públicas. Ejercicios como las encuestas deliberativas, los consejos de barrio o de distrito, los círculos de estudio o los presupuestos participativos, entre otros, se han puesto en marcha en municipios de diferentes lugares del mundo con el fin, en general, de profundizar la democracia local.

Recientemente, la literatura ha tratado de identificar las variables estructurales que afectan a los resultados de los procesos participativos puestos en marcha y su capacidad real de profundizar la democracia local. El capital social ha sido considerado en algunos estudios como factor determinante del rendimiento de los mecanismos de participación (Baiocchi et al., 2011; Avrtizer, 2010; Brugué et al., 2003; Walliser, 2003). Aún sin generar consenso sobre sus efectos positivos en la participación ciudadana, la literatura sobre esta materia ha aludido continuamente al capital social como una variable clave. La habilidad de cooperar con otros individuos para alcanzar metas colectivas, la confianza en los demás, las relaciones de reciprocidad en una comunidad y la generación de normas compartidas y respetadas por todos son aspectos que, según algunos autores, facilitan los procesos participativos y suscitan en los individuos el interés por los problemas públicos y la intervención en la esfera pública. En general, en estos estudios se considera el capital social como un equivalente de la noción de comunidad cívica, propuesta por Putnam (1993).

Tomando la acción colectiva como común denominador, Putnam intentó articular los postulados de la tradición de la cultura política y las nuevas corrientes institucionalistas, abordando preocupaciones compartidas por ambos enfoques teóricos (Jordana, 2000; Mota y Subirats, 2000; Levi, 1996). Su incorporación de elementos como la confianza, la reciprocidad y las formas de participación civil para la comprensión de los procesos de acción colectiva, llevó a identificar cómo estos resultan centrales para lograr la cooperación social (Ostrom y Ahn, 2003: 157). Putnam (1993; 2000) señala que los vínculos horizontales, derivados entre otros de las asociaciones civiles, promueven lazos de confianza, reciprocidad y cooperación, los cuales, a su vez, generan actitudes ciudadanas orientadas hacia lo público. El anterior círculo virtuoso fomenta una comunidad cívica, interesada en el bienestar general y en la participación en asuntos públicos, así como ciudadanos comprometidos con su comunidad. Este planteamiento y su énfasis hacia las asociaciones civiles y, por ende, hacia el aspecto de las redes, será retomado a menudo para explicar los efectos de del capital social en los rendimientos y resultados de las experiencias participativas.

A pesar del uso frecuente del concepto de capital social en las investigaciones sobre participación ciudadana en gobiernos locales, existe una notable heterogeneidad tanto 
respecto de su significado y operacionalización, como de sus relaciones causales. El presente artículo trata de contribuir a este debate teórico a partir de una revisión crítica de la literatura. Para ello, el artículo se estructura en dos apartados. El primero explora el concepto de capital social a fin de identificar sus diferentes acepciones, así como los principales enfoques en referencia a las relaciones micro y macro que establecen y, en particular, abordando la relación entre capital social y participación ciudadana. El segundo apartado presenta los principales debates respecto a la operacionalización del capital social, exponiendo cómo han observado esta variable los estudios empíricos de participación local, para finalizar con una discusión acerca de posibles alternativas. Esto último se hace a partir de aportaciones desde la literatura de community studies, el urbanismo y la teoría de redes, cuyos autores también se ocupan de la observación del contexto local.

\section{HACIA LA COMPRENSIÓN DEL VÍNCULO ENTRE CAPITAL SOCIAL Y PARTICIPACIÓN CIUDADANA}

\section{Debates en torno al capital social: conceptos y teorias}

El concepto de capital social ha suscitado importantes debates académicos aún irresueltos. Ha pasado de ser un concepto «sombrilla», aún emergente, a convertirse en un término cotidiano en diferentes campos del conocimiento (Woolcock, 2010). A pesar de su amplia difusión y de su éxito notable, son continuas las críticas acerca de la utilidad teórica del concepto y de su uso operativo en estudios empíricos. Para Woolcock (1998), no solo se trata de un término que ha tenido un uso indiscriminado, aplicado a diferentes problemáticas sin una adecuada rigurosidad, sino que resultado de lo anterior, se ha generado una dispersión de significados que más que contribuir a su desarrollo, ha supuesto un obstáculo para su consolidación teórica.

Haciendo una revisión de la literatura teórica sobre el tema se observa que, en general, las investigaciones han abordado tres dimensiones del capital social: las redes, la confianza y las normas sociales. Este abordaje ha sido heterogéneo, ya que algunos enfoques teóricos se han centrado en determinados aspectos específicos, como es el caso de la teoría de redes, y otros han realizado análisis más comprensivo de las tres dimensiones. De igual forma, parte de literatura sobre el tema se ha interesado más en los efectos individuales de este recurso, mientras otros, como se verá, han privilegiado sus consecuencias colectivas.

El concepto de capital social fue retomado desde la teoría de redes, desarrollada por la sociología y la economía, partiendo de la afirmación de que las redes sociales importaban. Esta cuestión llevó a acuñar la frase que será emblemática de la teoría del capital social, «no es lo que sabes o conoces sino a quién conoces» (Narayan y Woolcock, 2000: 1). Desde esta perspectiva, la relevancia del capital social, y con ello de las redes sociales, reside en su capacidad para generar recursos que pueden ser aprovechados por los individuos. Para Lin (2001) el capital social refiere a los recursos 
incorporados en las redes sociales, a los cuales los sujetos pueden acceder o movilizar a través de los vínculos existentes en tales redes. Este planteamiento va a constituir un primer enfoque dentro de las teorías de capital social, que destacan los efectos individuales de la pertenencia a redes sociales. Sin estar estrictamente vinculado a la teoría de redes, Bourdieu va a realizar aportaciones a la comprensión de la dimensión individual al capital social al subrayar la relevancia y los beneficios potenciales que obtienen los sujetos que participan en grupos, definiendo el capital social como: «El agregado de los recursos reales o potenciales que se vinculan con la posesión de una red duradera de relaciones más o menos institucionalizadas de conocimiento o reconocimiento mutuo» (1985: 248).

El planteamiento de Coleman $(1990 ; 1988)$, por el contrario, busca integrar los tres aspectos centrales del capital social: las redes, la confianza y las normas. Efectúa una transición micro-macro con el objeto de articular las dimensiones individuales y colectivas de este recurso. Así, para este autor, el capital social alude a los recursos subsecuentes de las relaciones sociales, que facilitan la interacción entre actores, y de ellos con la estructura social. A diferencia de otras formas de capital, como el humano y el físico, para Coleman este recurso no pertenece a los individuos, sino a la colectividad.

Para Ostrom, el capital social no solo es un acumulado de normas, saberes compartidos y reglas de uso, sino también un medio para solucionar problemas de acción colectiva. Las normas compartidas y patrones sociales generan capital social, y este recurso permite la construcción de arreglos institucionales para resolver dilemas vinculados a recursos de acervo común, evitando la generalización de estrategias individuales (Ostrom, 1990; Ostrom, 2000).

Por su parte, la formulación de Putnam, retomando el planteamiento de Coleman y a diferencia de la teoría de redes, destaca el carácter colectivo del capital social, cuya principal propiedad es la de facilitar la cooperación social. Los desarrollos en la literatura académica posteriores han explorado los vínculos entre cooperación, reciprocidad y confianza con diferentes fenómenos sociales, a fin de identificar cómo las relaciones sociales y la pertenencia a grupos y redes actúan para beneficio no solo de los sujetos, sino de la sociedad en su conjunto. Se trata, en general, de estudiar cómo el capital social permite superar la lógica de maximización individual y, por ende, los dilemas de la acción colectiva (Ostrom y Ahn, 2003).

La cuestión anterior, acerca de qué tipo de bienes produce el capital social, si individuales o colectivos, ha sido motivo de malentendidos y aplicaciones muy diferentes del concepto. Esto, a su vez, va a estar asociado a las discusiones sobre cómo operan las relaciones causales que llevan a identificar al capital social como variable explicativa de un sinnúmero de fenómenos sociales.

Como se señaló anteriormente, uno de los aspectos problemáticos en la consolidación teórica del capital social ha sido la heterogeneidad de significados que han surgido alrededor de este concepto. Tal diversidad conceptual ha tenido dos efectos. En primer lugar, ha obstaculizado el uso sistemático del capital social en investigaciones empíricas. De manera habitual los estudios en esta materia suelen hacer énfasis en los aspectos que estos consideran relevantes del capital social, obviando los demás. Así, se 
centran bien en las redes, bien en la confianza, bien en las normas sociales, dejando de lado análisis más comprehensivos que integren de manera simultánea los tres aspectos (Adam y Rončević, 2003). El segundo efecto derivado de la multiplicidad de acepciones tiene que ver con que cada definición entraña unas relaciones causales específicas asociadas al capital social. De esta manera, determinado concepto de capital social lleva aparejado una explicación concreta acerca de cómo opera este recurso y su vínculo con las realidades sociales que se busca comprender.

La acelerada expansión de los análisis basados en el capital social se ha debido a la multiplicidad de cuestiones que se pretenden explicar a partir de un planteamiento básico: las relaciones sociales tienen una capacidad potencial de generar beneficios tanto individuales como colectivos. La teoría de redes, las obras de Coleman, Ostrom y Putnam, así como los desarrollos posteriores, han tenido en común el interés de explorar de qué manera las conexiones sociales tienen efectos que van desde lo individual (micro), hasta lo colectivo (macro). Las diferencias surgen a partir de las relaciones causales que establece cada enfoque teórico y los alcances atribuidos a este recurso. A juicio de Castiglione et al. (2008: 4) el intento inaugurado especialmente por Putnam, de comprender cómo ciertos macroacontecimientos sociales son causados a partir de micromecanismos, vinculando las esferas micro y macro y conciliando con ello diversas tradiciones teóricas, ha causado cierta confusión respecto a la capacidad explicativa y a la parsimonia interna de la teoría del capital social.

Esser señala que es posible identificar dos formas de capital social: una de tipo más individual y otra más colectiva (Esser, 2008: 23). El capital social de tipo más individual, al que Esser denomina capital relacional, hace referencia a los recursos que un individuo puede utilizar como resultado de sus relaciones personales, tanto directas como indirectas. Cuestiones tales como el acceso a la información, la confianza al emprender tareas con otros actores, la solidaridad y el apoyo son algunos de los recursos y beneficios del capital relacional $(i d$.). La teoría de redes es uno de los exponentes más notables de esta corriente, cuya perspectiva micro busca identificar cómo las redes sociales entrańan recursos que pueden ser obtenidos o movilizados por actores individuales (Lin, 2001; Burt, 1992).

La relación causal que establecen los enfoques que estudian el denominado capital social relacional tiene que ver con la manera a través de la cual los individuos movilizan recursos derivados de las redes sociales con el objeto de obtener beneficios específicos, de allí que sea un capital que produzca bienes privados. Un ejemplo es el proceso de búsqueda de empleo, en el cual los individuos activan sus redes sociales, obteniendo información privilegiada a la que de otra manera no se accedería. Uno de los autores pioneros sobre esta temática es Granovetter (1974), quien analiza el papel de los lazos sociales en la búsqueda de empleo y encuentra que las redes sociales a las que pertenezcan los individuos pueden ser tan o más importantes que la formación académica. Si bien la teoría de redes, en particular las contribuciones de Granovetter, Lin y Burt, ha intentado vincular las relaciones micro con fenómenos sociales más amplios, en general este enfoque ha considerado el capital social como un recurso individual, del cual los sujetos hacen uso para su propio beneficio, y depende de la densidad de redes sociales a las que se pertenezca. 
A pesar de reconocer la dimensión individual del capital social, algunos autores han coincidido en que, a diferencia de otros tipos de capital como el humano o el económico, los recursos que se movilizan en las relaciones sociales, por una parte, no son controlados completamente por los actores individuales y, por otro, sus beneficios no recaen exclusivamente en los sujetos, sino que se difunden a través de toda la red social a la que se pertenece. Estos enfoques han sido agrupados por Esser bajo la denominación de capital social sistémico (2008: 37-41). Para Esser, a diferencia del capital social relacional, el sistémico no puede ser alcanzado por los esfuerzos ni la intención individuales, ya que se trata de un fenómeno colectivo que genera beneficios o recursos sociales tales como la confianza generalizada, la cooperación social, los valores y los principios morales compartidos. Otro elemento diferenciador, a juicio de Esser, es que mientras que en el capital relacional los actores afectados o beneficiados por la red social son aquellos que específicamente pertenecen a ella, en el caso del capital social sistémico se trata de una red social más amplia — por ejemplo, una comunidad, una región, un barrio- que independiente de la inversión que hagan los actores a título individual, todos los que pertenecen de alguna u otra manera a tal red se beneficiarán de ella.

A este respecto, Portes señala que mientras que el capital económico y humano pertenece a los sujetos que lo ostentan, el individuo posee el capital social en tanto forma parte de una red, pero son los otros los que sacan provecho de su pertenencia (1998: 7). Tal dualidad, el no control del capital y los beneficios dispersos a través de la red, han sido el origen de los enfoques denominados por Ostrom y Ahn (2003) maximalistas del capital social, que reconocen los efectos de las redes sociales en múltiples fenómenos sociales.

Coleman resalta que las tres formas principales que adopta el capital social son: a) las obligaciones, expectativas y credibilidad de las estructuras; b) los canales de información y, por último, c) las normas y sanciones efectivas (Coleman, 1988: 101-105). A juicio de Ostrom y Ahn (2003: 162-163), la contribución de Coleman puede ser considerada como una visión transicional entre la perspectiva minimalista de la teoría de redes y la visión más expansionista que aborda el capital social como un recurso que hace posible resolver dilemas de acción colectiva.

Por su parte, al estudiar el cambio institucional a partir de la observación de las regiones del norte y sur de Italia, Robert Putnam y sus colegas llegan a la conclusión de que el rendimiento de las instituciones, en este caso democráticas, está condicionado por el contexto social en el que se hallen (1993). En ese orden, un mismo diseño institucional puede funcionar de forma distinta dependiendo del contexto en donde se encuentre, por lo que el éxito de las instituciones democráticas, más que del diseño en sí, obedece a las condiciones contextuales, en particular sociales, en las que opere. A juicio de los autores, gran parte de la gobernabilidad democrática y la prosperidad económica en las sociedades actuales se explica por la existencia de ciertos atributos dentro de la organización social, como la confianza, la reciprocidad, la cooperación y el acatamiento de normas y reglas, que facilitan las acciones coordinadas, es decir, por los acumulados de capital social existentes (ibid.: 198). Así, Putnam utiliza el término 


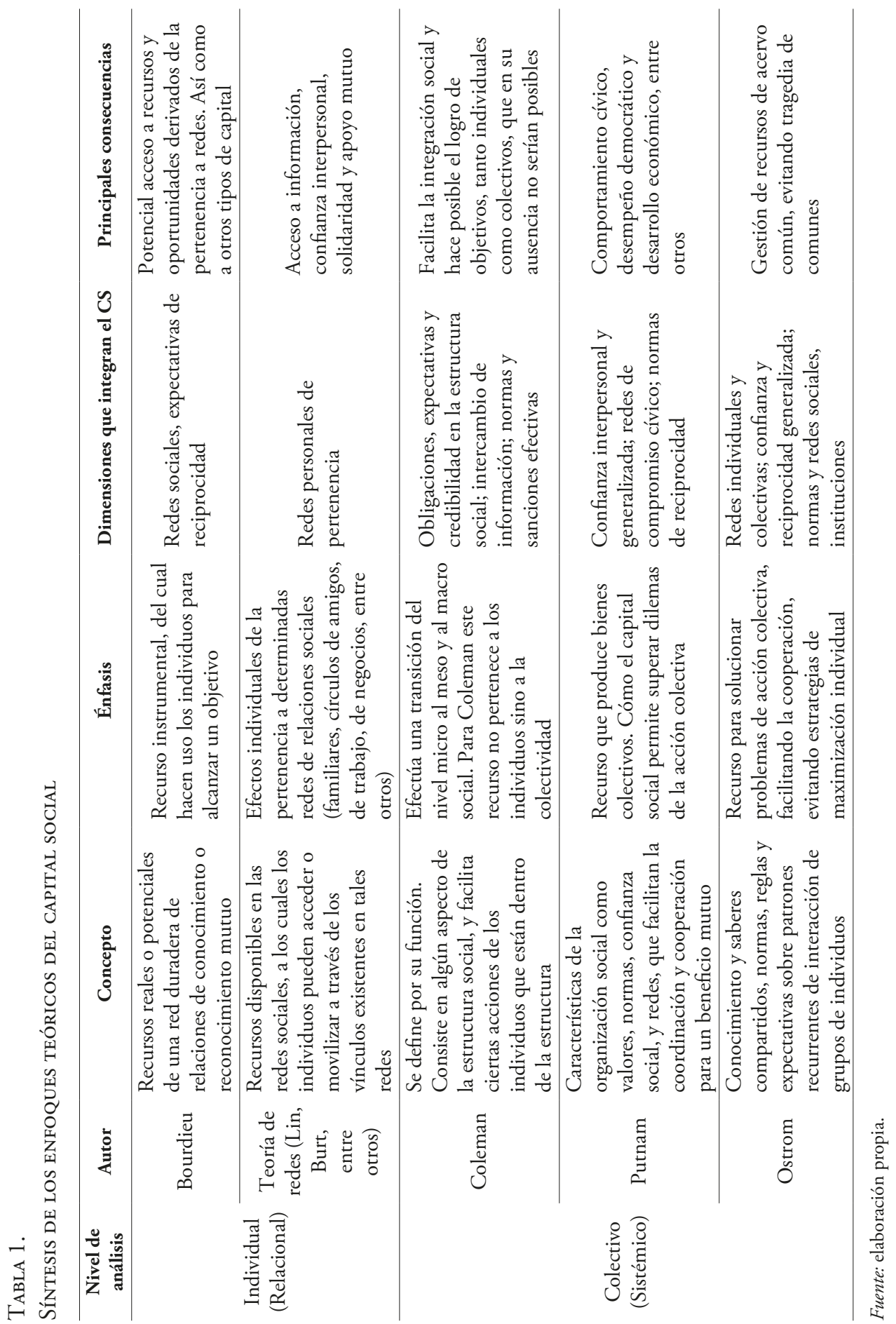


de capital social, empleado por Coleman y Bourdieu, dándole un nuevo significado y señalando que las redes sociales, los sistemas de reglas y normas creados a partir de acciones conjuntas, forman un recurso — capital social - que permite lograr metas que benefician a toda la comunidad. Ello redunda en un mejor desempeño democrático, reconociendo el valor para lo público de las actividades colectivas.

La perspectiva expansionista del capital social parte del planteamiento de que las conductas y actitudes generadas en el desarrollo de las relaciones sociales van estableciendo progresivamente un sistema de valores, normas y reglas compartidas, que a su vez generan sanciones sociales a aquellos que las incumplan, limitando así el comportamiento oportunista. Tales sanciones, así como la reciprocidad repetida, incrementan la confianza social y hacen posible la cooperación en gran escala. Por ello se destaca que el capital social está compuesto por redes, confianza y normas sociales. La tabla 1 resume los enfoques de capital social antes enunciados.

\section{Capital social. ¿Elemento clave para la participación ciudadana?}

Conforme han aumentado las iniciativas de participación ciudadana local en diversos lugares del mundo, han ido creciendo los intentos por examinar dichos procesos. Recientemente, el interés por identificar las variables que explican los desiguales rendimientos de los procesos participativos en los lugares que se ponen en marcha ha llevado a explorar cómo influyen las variables contextuales en el desarrollo y aportación a la democracia local de las experiencias participativas. Para autores como Lowndes y Pratchett (2009: 101), los instrumentos de participación que funcionan bien en un lugar no necesariamente funcionan bien en otro, lo que lleva a preguntarse sobre los factores de contexto que inciden en los procesos participativos y hacen que estos funcionen en términos de su eficacia en la contribución a la democracia.

Sobre esta cuestión existen numerosas propuestas referentes a variables de contexto, que resultan claves para explicar los rendimientos de los procesos participativos. Una de las variables que más ha sido retomada por diferentes estudios es el capital social (Vásquez, 2013; Baiocchi et al., 2011; Avrtizer, 2010; Velásquez y González, 2004; Walliser, 2003). La disposición de la comunidad a formar parte de los procesos participativos se traduce en la pluralidad del proceso, un mayor número de personas participando en él, mayor interés hacia lo público, calidad en las discusiones y articulación de visiones. La existencia de acumulados de capital social facilita la acción colectiva, permitiendo la movilización, la articulación de intereses y la deliberación.

Las aportaciones de Putnam, en particular su concepto de comunidad cívica, van a ser centrales para los estudios de participación ciudadana. La comunidad cívica es entendida como «aquella integrada por ciudadanos virtuosos que participan activamente en asuntos públicos, bajo condiciones de igualdad política, socializados en valores y actitudes cívicas» (Putnam, 1993:88). Se trata de comunidades cuyos ciudadanos cuentan con actitudes orientadas hacia lo público, que tienen la capacidad de articular intereses individuales en torno a un objetivo social y configuran lo que posteriormente 
Putnam denominará virtud cívica. Para este autor «en la comunidad cívica, los ciudadanos persiguen lo que Tocqueville llama 'interés individual debidamente entendido' [...] definido en un contexto de necesidades públicas más amplias [...] donde el interés personal está en función de los intereses de otros» (id.).

A juicio de Putnam, la existencia de una comunidad cívica activa se traduce en un mayor control de las acciones de gobierno (accountability). El autor parte del supuesto de que un buen gobierno será aquel que es más receptivo a las demandas ciudadanas (responsiveness), a la vez que opera con eficacia para atenderlas. Si este es el parámetro, ¿qué hace que una Administración sea más sensible a las necesidades de sus habitantes? La respuesta de este autor se centra en la capacidad de la ciudadanía en manifestar sus demandas y articularlas, lo que se logra a través de acción colectiva coordinada, es decir, capital social, cuya base será una comunidad cívica.

Las investigaciones de participación ciudadana que emplean la variable de capital social, retomando los conceptos de comunidad cívica y de virtud cívica, señalan que aquellas comunidades que cuenten con estas características y, en general, logren superar los dilemas de la acción colectiva, serán más proclives a participar de lo público, presionar por mayor accountability y responsiveness por parte de las autoridades de los gobiernos locales, dando como resultado procesos participativos más exitosos. De igual manera, es probable que las mismas comunidades presionen por gobiernos participativos o que en una vez puestos en marcha los procesos de participación los ciudadanos se involucren de manera activa en ellos.

En su estudio sobre casos exitosos de participación ciudadana, Fung y Wright (2003) destacan que uno de los elementos claves de las experiencias exitosas estudiadas tiene que ver con la capacidad de la ciudadanía para coordinarse y cooperar, así como con la práctica deliberativa. La deliberación, a juicio de estos autores, a su vez estará asociada con la existencia de una vida cívica y con organizaciones no gubernamentales que favorezcan una democracia vigorosa (ibid.: 40). Para ambos autores, una vida cívica activa permite la articulación de intereses y pasar del «yo» al «nosotros». En ese caso, las comunidades orientadas hacia el interés general, que conocen sus problemáticas, son funcionales a los procesos participativos, potenciando los resultados exitosos.

\section{MIDIENDO EL CAPITAL SOCIAL}

\section{Operacionalización del capital social: retos para la investigación}

Parte de las críticas a la teoría del capital social han estado orientadas a la debilidad de sus planteamientos teóricos, bien por el carácter tautológico de parte de sus explicaciones o por la inconsistencia al vincular los micromecanismos bajo los que opera el capital social con los macrofenómenos sociales que busca analizar. En términos de Van Deth (2003: 80) la teoría en este campo parte de suponer que los recursos de capital social tendrán como resultado una disminución de los costos de transacción de todos los participantes en una determinada red debido a que las relaciones de confianza 
generan que se necesiten menos recursos para garantizar cumplimiento. Sin embargo, no queda claro cómo conectar de manera convincente la manera en la que la confianza interpersonal impacta en el funcionamiento de la democracia o en la pobreza. En ese sentido, hay claridad sobre cómo las redes individuales producen bienes y beneficios a los individuos que pertenecen a dicha red; sin embargo, tal claridad pierde consistencia cuando se intenta conectar el funcionamiento de tales redes individuales con fenómenos macrosociales.

A fin de verificar las hipótesis derivadas de la teoría, en este caso del capital social, es preciso operacionalizar los conceptos. Sin embargo, como se apreció en el apartado anterior, existe una amplia diversidad de acepciones, y ello conduce necesariamente a que la operacionalización del capital social sea igualmente heterogénea. Como ya se ha señalado en el apartado anterior, tres han sido las propiedades básicas que tradicionalmente se han atribuido al capital social y sobre lo cual hay un consenso relativo: redes, confianza y normas. Cada una de estas propiedades, a fin de ser operacionalizadas, puede ser observada tanto en una dimensión individual como colectiva.

En el plano individual la manera de medir las redes sociales suele ser a través de encuestas o polling methods que indagan sobre el grado de membresía a asociaciones civiles, la pertenencia a redes y el grado de contacto social. En la dimensión colectiva se suelen observar estos mismos indicadores pero a nivel agregado ${ }^{1}$ (ibid.: 160). A pesar de que estos métodos han sido tradicionalmente aceptados, algunos autores son críticos con los datos existentes y señalan que el diseño adecuado de las encuestas es crucial para medir de manera apropiada la participación en asociaciones a fin de lograr afirmaciones teóricas más sólidas² .

Al tratar de medir aspectos como la confianza y las normas sociales los investigadores se enfrentan a un terreno mucho más difícil, en particular cuando el reto consiste en encontrar indicadores para la dimensión colectiva de tales aspectos (Inkeles, 2000). Para la medición de la confianza y las normas a nivel individual se utilizan frecuentemente encuestas o polling methods, mientras que en el plano colectivo se incorporan indicadores agregados. Sin embargo, algunos autores han cuestionado esta práctica porque se cae en el riesgo de utilizar datos del nivel micro, derivados de encuestas, para comprender fenómenos macro sin que haya claridad en esta conexión. A este respecto, Van Deth se pregunta: « Los datos agregados de la encuesta sobre confianza individual miden realmente la cantidad de confianza disponible como un bien

1. A este respecto, Van Deth (2008) realiza una contribución importante al clasificar las diferentes estrategias de investigación que corresponden a cada una de las propiedades del capital social: redes, confianza y normas. A su juicio, las redes corresponden al aspecto estructural del capital social, mientras que la confianza y las normas tienen que ver con aspectos más culturales.

2. Morales (2004) resalta, tras analizar tanto encuestas en Estados Unidos como el Eurobarómetro, que abordan la participación en asociaciones civiles, que la mayoría de indicadores que miden el grado de compromiso en estas organizaciones no son muy diferentes a los diseńados por Almond y Verba en 1963. Por otra parte, existe, en general, una ausencia de encuestas específicamente diseñadas para recoger información y datos sobre la participación en asociaciones. 
colectivo para todos los ciudadanos? ¿Qué se mide si simplemente contamos el número de asociaciones voluntarias de cada encuestado y calculamos el promedio de membresía en asociaciones voluntarias en una sociedad?» (2008: 161-162).

Si bien la información que ofrecen las encuestas para identificar los diferentes aspectos del capital social — redes, confianza y normas - resulta valiosa, especialmente en el plano individual, algunos autores reclaman la necesidad de ampliar las estrategias de investigación. En particular, aspectos como la confianza y las normas sociales en la dimensión colectiva, cuya medición resulta más compleja, pueden ser abordados haciendo uso de observaciones directas o estudios en profundidad de las comunidades (ibid., 2008: 160).

A este respecto, Van Deth $(2003 ; 2008)$ hace algunas precisiones y propuestas relevantes para los estudios que utilizan como variable el capital social. En primer lugar, señala que siguiendo el enfoque establecido por Coleman y Putnam, el capital social ha sido usualmente definido por sus funciones, por lo que la manera en que se manifiesta y los indicadores utilizados en cada caso serán diferentes. Así, teniendo en cuenta que las funciones del capital social cambian dependiendo de los diferentes contextos y fenómenos que buscan ser analizados, la definición que utilizar en cada caso derivará de la situación específica en la que se halle.

La plasticidad del concepto de capital social, a juicio de Van Deth puede dar lugar a diseños de investigación más robustos en este ámbito, pero conlleva romper esquemas ortodoxos (2003: 80-84). Esto es así debido a que, en vez de continuar con el debate respecto a hallar una definición categórica del concepto siguiendo una estrategia topdown, se precisa de una aproximación bottom-up que reconozca que el capital social es un término vinculado a la función que desempeña. Dado que su significado dependerá de la situación específica que se busca estudiar y del contexto en el que se enmarque, Van Deth sugiere, cuando se emprende una investigación sobre este tema, empezar por revisar las acepciones existentes de capital social y determinar sus rasgos comunes a fin de definir los aspectos necesarios para describir las características básicas que entrañan los diferentes conceptos de capital social. Una vez se establecen los rasgos fundamentales del capital social que se encuentran asociados al fenómeno concreto para analizar, se procede a definir la correspondiente estrategia de investigación (2008: 154).

\section{Capital social, asociacionismo y participación ciudadana. Los problemas de operacionalización}

La mayoría de las investigaciones sobre participación ciudadana local que hacen uso de la variable capital social, operacionalizan este concepto a través de indicadores que miden el grado de membresía en asociaciones voluntarias. Esto se puede explicar debido a la utilización del enfoque de Putnam en muchas de las investigaciones y de su concepto de comunidad cívica. Putnam señala que las asociaciones civiles serán fundamentales en la reproducción y potenciación de los acumulados de capital social de una sociedad, tanto a nivel interno, en los individuos, como externo, en los 
procesos de gobierno. En el plano individual, las asociaciones internalizarán en el comportamiento de los ciudadanos hábitos de cooperación, solidaridad y espíritu cívico $^{3}$ (Putnam, 1993: 86-91). Respecto a su efecto externo, las asociaciones facilitarán la articulación de intereses, o que posibilitará una colaboración social efectiva, confianza social y normas de reciprocidad generalizadas (ibid.: 163-170). En ese caso, se puede suponer que si existen ciudadanos cívicos y comunidades en donde prevalezca el interés por lo público, la cooperación y la confianza generalizada es probable que los procesos participativos sean más exitosos. Y ¡cómo medir la existencia comunidad cívica y virtud cívica? La respuesta de Putnam es que a través de asociaciones civiles. Debido a este planteamiento, buena parte de los estudios sobre participación, que emplean como variable el capital social, operacionalizan dicho concepto mediante indicadores de membresía y actividad en asociaciones civiles.

Dentro de la literatura de capital social no solo se ha constatado los efectos positivos de las asociaciones en los individuos, por ejemplo, al promover la participación y discusión políticas (Verba et al., 1995); también se ha considerado los beneficios de las asociaciones a nivel más macro, entre otras, como escuelas de democracia (Cohen y Rogers, 1995). Las asociaciones civiles ejemplifican la superación de dilemas de acción colectiva al promover la cooperación y la coordinación social. Como consecuencia, sus potenciales efectos pueden ser la confianza interpersonal, la solidaridad y el apoyo mutuo, además de servir como canalizadores de intereses y demandas sociales. Por ello han sido elementos centrales en investigaciones sobre democracia y, este caso, participación ciudadana en gobiernos locales.

Son diversos los estudios que establecen la relación positiva entre asociacionismo y rendimiento de prácticas participativas en gobiernos locales. Un ejemplo es la investigación de Wampler y Avritzer (2004), quienes ponen de relieve cómo las estrategias adoptadas por organizaciones de la sociedad civil durante el proceso de transición en Brasil promovieron la creación de instituciones participativas locales, entre ellas el presupuesto participativo. Los autores seńalan que los resultados de los presupuestos participativos, en términos de implementación de proyectos seleccionados así como capacidad de deliberación y negociación de los asistentes a estos espacios, dependen en buena medida de la tradición asociativa (ibid.: 308-309). En aquellas ciudades o barrios dentro de la ciudad con mayor número de organizaciones de la sociedad civil se aprecia una participación basada en lo que denominan deliberación "cara a cara», en la capacidad de movilización y presión para implementar los proyectos acordados y evitar la intermediación clientelar.

En relación con la pregunta sobre cuáles son las condiciones adecuadas para que las instituciones participativas locales tengan éxito, y a partir de un estudio comparado en diversas ciudades de Brasil, Avritzer (2010), señala que las características organizativas

3. Putnam corrobora este planteamiento a partir de las evidencias encontradas por Almond y Verba en su libro The Civic Culture, que revelan la relación entre membresía asociativa y la confianza social, la participación política y la competencia cívica subjetiva (Putnam, 1993: 90). 
de la sociedad civil serán esenciales para garantizar el éxito de los procesos participativos. Más que centrarse en el capital social en su conjunto, nuevamente recurre a las asociaciones y los patrones asociativos para explicar los rendimientos de los procesos participativos. A partir del porcentaje de población afiliada a asociaciones o el número de asociaciones vecinales explora las características asociativas en los distintos lugares analizados.

En la misma línea de identificar factores que desempeñan un papel central en el rendimiento de espacios de democracia participativa local, Font y Galais (2011) destacan que variables como la ideología política de los partidos que llevan a cabo el proceso participativo, el apoyo externo y la sociedad civil resultan claves para comprender los resultados en términos de calidad democrática de estos procesos. A juicio de estos autores, en caso de que la sociedad civil, en su dimensión organizativa, esté presente en la iniciativa o promoción del proceso participativo (bottom-up) hará que éste sea más inclusivo y deliberativo (ibid.: 12).

Haciendo una lectura crítica de la literatura que aborda la relación positiva entre sociedad civil y democracia, Baiocchi et al. (2011) proponen una tipología para comprender la relación entre sociedad civil y Estado para explicar los rendimientos de los procesos participativos locales. A juicio de los autores, es preciso identificar las características de la sociedad civil en términos de su capacidad de autoorganización y la manera cómo los actores sociales se vinculan con el Estado a fin de hacer efectivas sus demandas. Así, una sociedad civil autónoma, que es capaz de generar procesos autoorganizativos de manera independiente, así como de determinar de forma propia sus demandas y que se vinculen con el Estado en el marco de reglas claras -institucionalizadas-, es probable que genere una democracia movilizada y prácticas participativas que fortalezcan la deliberación y la participación plural, evitando patrones clientelares (ibid.: 33-38).

Las investigaciones de Wampler y Avritzer (2004), Avritzer (2010), Font y Galais (2011) así como Baiocchi et al. (2011), reflejan la tendencia en los estudios de participación ciudadana local de insertar el término sociedad civil para hacer alusión, de manera amplia, a las características asociativas de la sociedad analizada, dejando de lado el probablemente problemático concepto de capital social. Estos estudios se basan, en general, en encuestas o entrevistas a líderes asociativos o miembros de asociaciones. Así, dichas investigaciones evidencian el énfasis en el nivel individual de la dimensión relativa a las redes del capital social, eludiendo aspectos como la confianza y las normas tanto en su nivel individual como colectivo.

Este énfasis hacia el carácter organizativo y formal de las redes si bien permite hacer un acercamiento a las características del capital social de determinada comunidad, es limitado por varias razones. En primer lugar, las investigaciones sobre participación política han expuesto el estrecho vínculo entre recursos individuales y probabilidad de pertenencia a asociaciones civiles (Verba et al., 1995); por ejemplo, las personas con mayores recursos socioeconómicos tendrán una probabilidad más alta de asociarse. Las diferencias de pertenencia no son solo socioeconómicas, también la raza y el género inciden en la participación en asociaciones. En el caso de las mujeres, si 
bien las cifras varían de país a país, en general se encuentra que el nivel de pertenencia a asociaciones es menor comparativamente al caso de los hombres, diferencias que se agudizan en el caso de que las mujeres tengan hijos pequeños ${ }^{4}$ (Norris y Inglehart, 2006). En el caso de la raza (Lin, 2000), algunos estudios han demostrado que la pertenencia a determinado grupo étnico o racial minoritario puede tener consecuencias negativas en formar parte activa de asociaciones civiles. Si estas diferencias son veraces, ¿qué pasa con aquellos individuos que por su raza, género o recursos socioeconómicos tienen una baja probabilidad de pertenencia a asociaciones? ¿Se puede llegar a la conclusión, en caso de que se trate del único indicador de capital social utilizado, de que estos grupos sociales no cuentan con capital social? Como críticos del capital social han destacado, este planteamiento teórico ha subestimado la existencia de relaciones de sociales y de poder desiguales que, entre otras, se manifiestan en la pertenencia a asociaciones, desatendiendo, por otra parte, la manera en que los grupos en desventaja generan relaciones de confianza, reciprocidad y cooperación.

En segundo lugar, como ya lo señalaba Putnam en su estudio sobre el asociacionismo en Estados Unidos (2000), el nivel de pertenencia a asociaciones ha bajado en las últimas décadas. Por otro lado, la pertenencia a asociaciones presenta una distribución desigual en las diferentes regiones del mundo ${ }^{5}$, pero aún en regiones cuyo asociacionismo parece ser relativamente alto no se aprecia necesariamente un vínculo positivo entre este indicador de capital social y el fortalecimiento democrático (Roßteutscher, 2008). Probablemente como respuesta a esto o debido al interés de explorar nuevos indicadores que den cuenta del capital social en diferentes sociedades sin reducir este recurso al asociacionismo, se han hecho esfuerzos por recolectar información sobre diferentes aspectos de la sociabilidad tanto formal como informal. Como señalaba el apéndice metodológico de la plataforma CIVICUS ${ }^{6}$ «existe un acuerdo general de que las organizaciones voluntarias son solo un pequeño grupo dentro de un entorno mucho más amplio, ya no pueden ser tratadas como representantes claves para todo el universo de la sociedad civil [...]. Esto ha hecho que muchas metodologías convencionales de simple reconocimiento del sector voluntario sean obsoletas para quienes

4. Lowndes (2003) destacaba que hombres y mujeres pueden estar involucrados en diferentes circuitos de capital social. En particular, redes de sociabilidad informal — como las redes de apoyo mutuo, contacto constante con familiares y amigos, así como redes vecinales de sociabilidad informal, entre otras - son importantes en el caso de las mujeres. Por lo anterior, Lowndes cuestiona el énfasis hacia la membresía en asociaciones como indicador predominante de capital social: «Hacer referencia a los 'niveles' agregados de capital social o elegir una sola medida (típicamente membresía asociativa), puede dar una imagen engańosa con respecto a las diferencias de género. En segundo lugar, las medidas cuantificables del capital social [...] pueden oscurecer importantes diferencias de género en el tipo o la calidad del capital social involucrado. En tercer lugar, la elección de medidas de capital social puede reflejar un sesgo de género, seleccionando dentro o fuera de las actividades dominadas por mujeres y hombres, respectivamente» (ibid.: 9).

5. Ver World Values Survey (http://www.worldvaluessurvey.org/WVSOnline.jsp).

6. Ver CIVICUS: World Alliance for Citizen Participation (https://www.civicus.org/). 
realmente están interesados en capturar la diversidad de la sociedad civil y cómo en última instancia contribuye a la democracia y el desarrollo» (Fioramonti y Kononykhina, 2013: 1).

En tercer lugar, la existencia de asociaciones civiles no supone necesariamente la presencia de una comunidad cívica en los términos presentados por Putnam. Estudios como el de Molenaers (2005) muestran que en países en vías de desarrollo los patrones asociativos no responden a las características mayoritariamente expuestas en la literatura sobre capital social. Es decir, no hay relación automática entre asociacionismo y confianza, reciprocidad y cooperación horizontal (ibid.: 164). Por el contrario, en muchos casos lo que se observa es que la vida asociativa está relacionada con patrones de interacción clientelista en donde los individuos buscan conseguir recursos sociales a través de líderes locales, quienes juegan un rol de intermediación entre los habitantes y los gobiernos locales, perpetuando el clientelismo. Sin embargo, es a través de redes informales en donde se pueden encontrar procesos de cooperación horizontal, confianza y reciprocidad.

Por último, existen nuevas formas de acción colectiva o nuevos activismos urbanos, como los denomina Walliser (2013). Estos grupos orientados hacia la producción de espacio urbano y la promoción de modos innovadores de participación ciudadana se caracterizan por su bajo nivel organizacional —a diferencia de formas tradicionales como las asociaciones de vecinos-, inteligencia colaborativa a través de redes sociales, y grupos más abiertos, flexibles y descentralizados. En definitiva, las tecnologías de la información y las comunicaciones (ICT en inglés) contribuyen a generar nuevos patrones de movilización y redes sociales entre ciudadanos (ibid.: 329), lo cual se escapa a la lógica de las redes formales, asociaciones y al ámbito tradicional de los estudios de participación. En esta misma línea, Savage et al., (2005) señalan que las nuevas formas urbanas desalientan el asociacionismo tradicional, y en ese nuevo marco espacial la organización social no requiere interacción social cara a cara.

Partiendo de las limitaciones antes señaladas, el próximo apartado buscará proporcionar herramientas analíticas alternativas para medir el capital social, especialmente en estudios de participación ciudadana en gobiernos locales.

\section{Capital social y participación ciudadana: Más allá de las asociaciones civiles}

Si se considera que el capital social alude a los patrones de sociabilidad, tanto formal como informal —en términos de redes, confianza y normas—, que facilitan la acción colectiva y la resolución de problemas comunes, al elegirla como variable dentro de un diseño de investigación sería adecuado incluir indicadores que no solo hagan énfasis en una de sus dimensiones, sino que aborden este recurso de manera comprehensiva $\mathrm{y}$, asimismo, tengan en cuenta sus aspectos formales e informales. Siguiendo a Van Deth (2008), la gran heterogeneidad conceptual del capital social no se refleja cablamente en su uso en términos empíricos. 
En general, la manera de abordar el capital social desde la ciencia política ha estado centrada en las asociaciones y la confianza individual. Por ello, es preciso aludir a nuevas aproximaciones teóricas que desde otras disciplinas puedan favorecer la producción de un conocimiento más robusto sobre el capital social y la cuestión participativa. Existen tres cuerpos de literatura que pueden contribuir al debate para pensar en diseños de investigación alternativos, bajo nuevas propuestas de operacionalización. Los denominados community studies, así como los estudios urbanos y el análisis de redes tienen una larga tradición de análisis del capital social que, sin embargo, ha sido retomada de manera muy tangencial por las investigaciones de participación local.

A pesar de la heterogeneidad que podemos encontrar en estos campos disciplinares, es posible identificar una preocupación común al estudiar el capital social, en particular en los community studies y en los estudios urbanos, y es la necesidad de considerar los contextos espaciales en los cuales se enmarca y se reproduce este recurso. En efecto, autores como Blokland y Savage (2008) señalan que muy a menudo las formas de medir el capital social se abstraen del lugar en donde este se enmarca, proponiendo indicadores abstractos que dan lugar a interpretaciones descontextualizadas que no comprenden cómo se forma y cómo está cambiado este recurso. Siguiendo a autores como Lefebvre, subrayan que las relaciones sociales son producidas a través de la organización del espacio. Así, será este el que permitirá comprender las dinámicas interrelaciones que generan el capital social de una determinada comunidad (ibid.: 7-8).

A partir de análisis históricos y etnográficos en dos ciudades, Blokland y Rae (2008) exploran el cambio que se está produciendo en las relaciones urbanas y cómo afecta esto a la naturaleza del capital social, entre esto a las redes sociales. A su juicio, las ciudades han cambiado y con ello la forma de crear capital social. Del localismo más amplio, que promovía la creación de asociaciones, se ha dado paso a formas más excluyentes, ejemplificadas en barrios aburguesados cerrados, pero no solo, ya que también hay guetos que han generado lo que los autores llaman la privatización de la comunidad, caracterizada por redes, pero también confianza y normas, más individualizadas y fragmentadas. Sin embargo, también han surgido relaciones en red donde el entorno local no es el enclave central de la socialización y donde las tecnologías de comunicación modernas pueden facilitar este proceso, generando comunidades y formas de socialización diferentes a las tradicionales

Un segundo punto que destaca en las áreas disciplinares antes mencionadas es su interés por las redes informales y su contribución para comprender procesos de coordinación social, confianza y configuración de normas sociales. A fin de explicar cómo influyen las características al interior de las redes individuales en la creación de capital social que facilita el compromiso político, Lake y Huckfeldt (1998) hacen un estudio de redes egocéntricas a partir de datos de los individuos y sus redes. Para los autores, el tamaño de la red, la frecuencia en la interacción y el conocimiento político de los participantes de la red determinan el nivel de compromiso político individual. No obstante, a su juicio el capital social no lo poseen los individuos, sino que se produce a 
través de patrones estructurados de interacción social, que la pertenencia a asociaciones por sí sola no consigue predecir. Es el funcionamiento de las redes informales lo que permite comprender tal proceso.

Por otra parte, en su estudio del papel del capital social en la participación democrática en el norte de la India, Krishna (2002) reconoce la limitación del asociacionismo como indicador de capital social en los casos de estudio, toda vez que el porcentaje de pertenencia en la zona es realmente bajo, del $5 \%$. Sin embargo, para el autor, no por esto se puede llegar a la conclusión de que no existe este recurso (ibid.: 446). A su juicio, los habitantes de los casos de estudio efectivamente cooperan entre sí en diferentes tareas, pero lo hacen a través de redes informales. Por lo anterior Krishna elaboró indicadores, para ser empleados en un análisis cuantitativo de encuestas, que consideran el tipo de actividades en las cuales los individuos en estas áreas cooperan. El autor identificó seis actividades que permitieron medir el funcionamiento y fortaleza de las redes locales, las normas relacionadas con la solidaridad, la reciprocidad y la confianza. Redes de trabajo colectivo y compartido, redes de ayuda en caso de contingencias, compromiso de los líderes con la comunidad, incluso el corregir conductas de niños que no pertenecen a la propia familia a fin de que cumplan las normas sociales. Estos indicadores propuestos son interesantes, ya que tienen en cuenta las particularidades de patrones de sociabilidad y cooperación en las zonas estudiadas.

Un tercer punto se refiere a cómo las desigualdades estructurales se reflejan en la configuración del capital social. Como se adelantaba en el apartado anterior, algunos autores han hecho una lectura crítica del capital social, en particular sobre la falta de atención prestada a las desigualdades estructurales que pueden persistir en las redes sociales. Si bien Bourdieu en sus planteamientos sobre capital social ya había destacado este asunto, pronto fue dejado de lado por la perspectiva de Coleman y Putnam. Autores como Haynes y Hernandez (2008) retoman el enfoque de Bourdieu para analizar cómo la raza y la existencia de un contexto históricamente segregado determinan las características del capital social. Asimismo, indagan la manera en que se afirman intereses colectivos de estos grupos para incidir en las decisiones de los gobiernos locales. A través de un análisis histórico, apoyado en entrevistas y revisión de archivo, los autores identifican que la comunidad negra en Runyon Heights (Estados Unidos) contaba en la primera mitad del siglo xx tanto con redes formales como informales de socialización muy importantes para mantener la cohesión social de su comunidad. Sin embargo, esto nos les aseguró que sus demandas fueran escuchadas por el gobierno local que para ese entonces estaba monopolizado por representantes de raza blanca, evidenciando una segregación y una exclusión institucional. Para estos autores, la manera que encontraron de influir en el gobierno fue a través de redes informales, contactos claves que trabajaban en el gobierno, no mediante sus asociaciones.

Por su parte, Lowndes (2003) explora cómo las características del capital social pueden ser útiles para explicar las diferencias en los patrones de participación política entre hombres y mujeres, particularmente en la política local. A partir de datos de la 
Encuesta General de Hogares británica (GHS), 2000-2001, que presenta cinco aspectos diferentes del capital social, la autora observó cómo los perfiles de capital social difieren para los grupos de encuestados en relación con el género. Estos datos, más otros obtenidos de investigación cualitativa, le permiten concluir que las mujeres tienen tanto capital social como los hombres, pero que es ligeramente diferente: su perfil de capital social está más orientado a redes de sociabilidad informal, especialmente en el contexto del vecindario. Algunos de los indicadores de la GHS, por ejemplo respecto a confianza y reciprocidad, indagan sobre el grado de cercanía, frecuencia en el contacto y ayuda a vecinos. Igualmente, preguntan sobre las redes de apoyo social, pedir un favor en caso de estar enfermo o pedir dinero en calidad de préstamo. Por último, la encuesta planea preguntas acerca de la participación cívica, si los individuos están informados sobre asuntos locales, si están involucrados en alguna organización local, o si se ha formado parte de tomar una acción concreta para resolver un problema local. De esta manera, la operacionalización del capital social contempla indicadores sobre redes, tanto formales como informales, pero también sobre confianza, reciprocidad y participación cívica.

Un último aspecto atiende la naturaleza conflictiva y tensa de las relaciones sociales que se refleja tanto en el funcionamiento de las redes como en la existencia o no de la confianza personal y social, así como en la manera en la que operan las normas sociales. Un estudio interesante, si bien no centrado directamente en el capital social, es el de Martínez y Lorenzi (2012), quienes a través de la investigación-acción participativa, en particular desde un proceso de investigación activista, estudian la experiencia del movimiento de ocupaciones en Madrid. Ya algunos autores han destacado la existencia de capital social en grupos que buscan dar solución a problemas habitacionales vía ocupación — squatters_- (Sengupta y Sharma, 2012). Lo interesante del estudio de Martínez y Lorenzi es la utilización de metodologías novedosas a fin de comprender cómo opera el movimiento de ocupación desde dentro, su organización interna, pero también sus conflictos, lo que contribuye a ofrecer una visión múltiple de este proceso. De igual forma, también pone en cuestión la manera a través de la cual, de manera convencional, se ponen en marcha mecanismos de participación ciudadana local, en su gran mayoría espacios institucionalizados que reproducen estructuras jerárquicas de poder, donde las autoridades locales y las élites condicionan en gran medida el proceso.

Por su parte, en su estudio comparado de participación ciudadana en dos ciudades con contextos sociales y políticos diferentes Vásquez (2013) analiza cómo los habitantes de zonas periféricas de las ciudades estudiadas se organizaron para dar respuesta a problemas habitacionales y de sus barrios, generando patrones diferentes de asociacionismo, pero también de la manera cómo se relacionan con el gobierno local. A partir de una revisión histórica y haciendo uso de entrevistas a informantes claves, identificó que la manera en la que se fue generando la acción colectiva, en términos de su autonomía y capacidad de auto-organización respecto a los gobiernos locales, generó patrones asociativos diferentes. En algunos casos más tendientes al conflicto, en otros a la cooperación y en otros al clientelismo. 
Woolcock y Nyham (2009) resaltan la necesidad de apostar por estrategias metodológicas mixtas al emprender investigaciones que incorporen como variable el capital social. A su juicio, buena parte de los estudios en este campo tienden a usar un único instrumento metodológico, bien sea de carácter cualitativo o cuantitativo. Por ello, los autores proponen instrumentos tanto cuantitativos como cualitativos que permitan operacionalizar esta variable. Respecto a los instrumentos cualitativos para medir redes sugieren hacerlo a través de preguntas tales como: cómo funcionan los grupos formales o informales, qué frecuencia de intercambio tienen, qué recursos se intercambian en dichas redes. En alusión a la confianza, las preguntas quieren averiguar a qué redes, grupos o instituciones formales o informales acuden los individuos para resolver problemas, cómo se distribuye la confianza en la comunidad (a través de la familia, a través de redes específicas o en las localidades en general). En referencia a la acción colectiva y la cooperación, plantean cuestiones sobre los ejemplos de acción colectiva llevados a cabo por la comunidad, cómo se inició, quiénes se movilizaron, cuáles son las sanciones sociales para quienes contravengan las normas sociales previstas. Los instrumentos cuantitativos que refieren a redes, además de las preguntas convencionales sobre membresía (pertenencia, número de personas que forman parte de la organización, frecuencia de interacción, características socioeconómicas, étnicas, religiosas de género de las personas que integran el grupo), también inquieren sobre si se acude a redes informales para pedir ayuda. Respecto a la acción colectiva y la cooperación, se pregunta si se ha participado en actividades para beneficio de la comunidad.

Finalmente, es de destacar la investigación de Lowndes et al. (2006) acerca de los factores que explican el funcionamiento de la participación local. A partir de una herramienta denominada $\mathrm{CLEAR}^{7}$ los autores establecen los aspectos que a su juicio son fundamentales para lograr una participación local exitosa. Una de las variables es capital social. A través de preguntas sobre el sentido de pertenencia a una comunidad, con las redes de confianza interpersonal existentes y la capacidad de cooperar de manera efectiva de los ciudadanos (lo que los autores denominaron like to), así como sobre las características y estructura asociativa de una comunidad, las redes y grupos sociales, en términos de asociaciones y capacidad de movilización (enabled to), los autos buscaron determinar el nivel de capital social en las distintas ciudades analizadas. Los métodos sugeridos fueron, además de estadísticas oficiales, información cuantitativa como encuestas. De técnicas cuantitativas se contempló el uso de instrumentos más cualitativos, como entrevistas (a políticos locales, a funcionarios que desempeñan tareas en áreas vinculadas a la participación ciudadana y a líderes sociales) y focus groups. Lo anterior da cuenta del diseño de métodos mixtos para estudiar el capital social. La tabla 2 ilustra las anteriores operacionalizaciones, efectuadas por los estudios

7. Lowndes et al., (2006) dirigieron un estudio a nivel europeo del European Committee on Local and Regional Democracy (CDLR), llevado a cabo en veintitrés municipios de cinco países, cuyo propósito era brindar una herramienta analítica para ayudar a los gobiernos locales a mejorar la comprensión de la participación ciudadana de sus municipios. 
Midiendo el capital social: una aproximación desde la participación ciudadana...

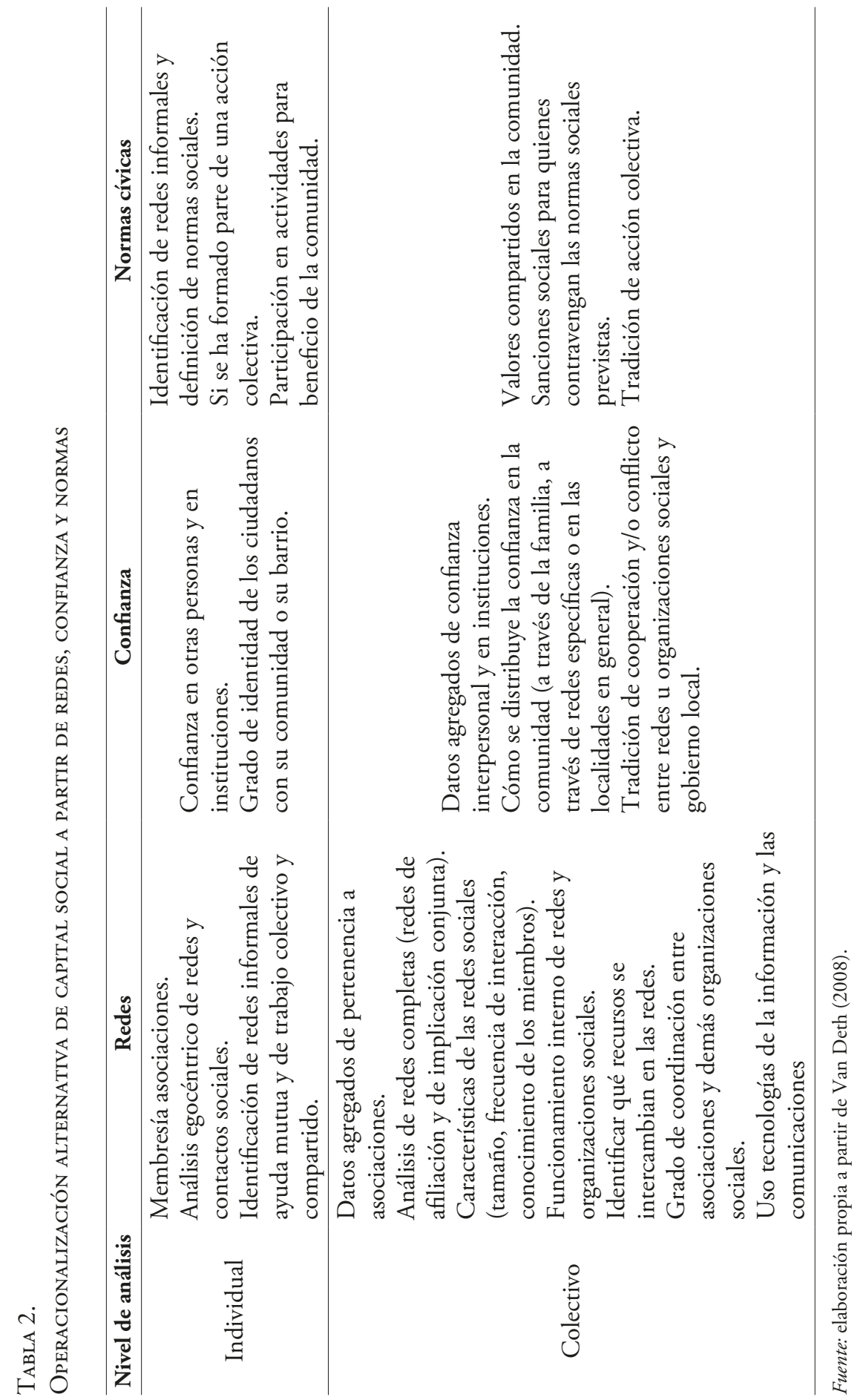

Revista Española de Ciencia Política. Núm. 48. Noviembre 2018, pp. 103-128 
expuestos, que abordan las redes, la confianza y las normas tanto desde el nivel de análisis individual como colectivo.

\section{CONCLUSIONES}

El capital social ha sido frecuentemente utilizado como una de las variables explicativas de los rendimientos de los espacios de participación ciudadana directa en gobiernos locales. Partiendo de los postulados de Putnam, se señala que los vínculos de confianza, reciprocidad y cooperación generan actitudes que fortalecen la democracia, en este caso, la local. Una vez aceptada dicha relación causal, diversos estudios empíricos han tratado de incorporar este factor como variable explicativa del fenómeno participativo local. En general, tales investigaciones han empleado el aspecto relativo a las redes, operacionalizando el concepto a través de la participación en asociaciones o número de asociaciones civiles. No obstante, si bien las asociaciones son un elemento central en los procesos democráticos, estudios recientes llaman la atención sobre la importancia de incorporar estrategias que permitan considerar el carácter multidimensional del capital social, así como el impacto del contexto en el cual se enmarca este recurso.

Explorando alternativas de operacionalización que den cuenta de la diversidad del capital social, en sus dimensiones de redes, confianza y normas, tanto a nivel individual como colectivo, se encuentran diversos aportes desde los community studies, los estudios urbanos y la teoría de redes que desde diferentes estrategias abordan la riqueza analítica de este concepto. Estos cuerpos de literatura tienen una extensa tradición de estudios sobre capital social que, no obstante, ha sido considerada de manera marginal en las investigaciones sobre participación. Sus contribuciones para operacionalizar la variable capital social pasan, entre otros, por analizar las características y el funcionamiento de las redes informales. De igual forma, pretenden identificar las desigualdades estructurales que afectan las diferentes dimensiones del capital social y su efecto en la participación local. Asimismo, analizan la naturaleza conflictiva que entrañan las relaciones sociales y el impacto del contexto en la configuración y desarrollo del capital social. En ese marco, proponen operacionalizaciones que proporcionan una visión más comprehensiva de la estructura, funcionamiento y características del capital social de una determinada comunidad. Más que una propuesta única de operacionalización, este artículo ilustra posibles alternativas. Puesto que el capital social es una variable que depende del contexto, cada investigador deberá considerarlo para diseñar una estrategia que se adecúe al caso de estudio.

\section{AGRADECIMIENTOS}

Agradezco los comentarios de Fabiola Mota (Universidad Autónoma de Madrid) y de Victoria Rivera (Universidad de Bristol). Igualmente, agradezco las excelentes 
sugerencias realizadas por el Consejo Editorial de la RECP, así como por los evaluadores externos y anónimos.

\section{Referencias}

Adam, Frane y Borut Rončević. 2003. «Social Capital: Recent Debates and Research Trends», Social Science Information, 42 (2): 155-83.

Avrtizer, Leonardo. 2010. Las instituciones participativas en el Brasil democrático. México DF: Universidad Veracruzana.

Baiocchi, Gianpaolo, Patrick Heller y Marcelo K. Silva. 2011. Bootstrapping democracy. Transforming local governance and civil society in Brazil. Stanford: Stanford University Press.

Blokland, Talja y Douglas Rae. 2008. «The End to Urbanism: How the Changing Spatial Structure of Cities Affected its Social Capital Potentials, en Talja Blokland y Mike Savage (eds.), Networked Urbanism. Social Capital in the City. Hampshire: Ashgate.

Blokland, Talja y Mike Savage. 2008. «Social Capital and Networked Urbanism», en Talja Blokland y Mike Savage (eds.), Networked Urbanism. Social Capital in the City. Hampshire: Ashgate.

Bourdieu, Pierre. 1985. "The Forms of Capital», en John G. Richardson (ed.), Handbook of Theory and Research for the Sociology of Education. Nueva York: Greenwood Press

Brugué, Quim, Joan Font y Ricard Gomá. 2003. «Participación y democracia: asociaciones y poder local», en María Jesús Funes y Ramón Adell (eds.), Movimientos sociales. Cambio social y participación. Madrid: Universidad Nacional de Educación a Distancia.

Burt, Ronald S. 1992. Structural Holes: The Social Structure of Competition. Cambridge Mass.: Harvard University Press.

Castiglione, Dario, Jan W. Van Deth, y Guglielmo Wolleb. 2008. "Social capital's fortune: An introduction", en Dario Castiglione, Jan W. Van Deth y Guglielmo Wolleb (eds.), The Handbook of Social Capital. Oxford: Oxford University Press.

Cohen, Joshua, y Joel Rogers. 1995. Associations and Democracy. Londres: Verso.

Coleman, James. 1988. «Social Capital in the Creation of Human Capital», American Journal Sociology, 94: 95-120.

Coleman, James. 1990. Foundations of Social Theory. Cambridge: Harvard University Press.

Esser, Hartmut. 2008. "The two meanings of social capital.» En Dario Castiglione, Jan W. Van Deth y Guglielmo Wolleb (eds.), The Handbook of Social Capital. Oxford: Oxford University Press.

Fioramonti, Lorenzo, y Olga Kononykhina. 2013. Methodological note on the CIVICUS' Civil Society Enabling Environment Index (EE Index). CIVICUS: World Alliance for Citizen Participation. Disponible en: https://bit.ly/2Os6F3e. 
Font, Joan (coord.). 2011. Democracia local en Andalucía. Experiencias participativas en los municipios andaluces. Sevilla: Fundación Pública Andaluza, Centro de Estudios Andaluces.

Font, Joan, y Carolina Galais. 2011. "The qualities of local participation: the explanatory role of ideology, external support and civil society as organizer», International Journal of Urban and Regional Research, 35 (5): 932-948.

Fung, Archon y Erik Olin Wright. 2003. Deepening Democracy: Institutional Innovations in Empowered Participatory Governance. Londres: Verso.

Granovetter, Mark. 1974. Getting a job. A study of contacts and careers. Cambridge, Mass.: Harvard University Press.

Haynes, Bruce D. y Jesus Hernandez. 2008. «Place, Space and Race: Monopolistic Group Closure and the Dark Side of Social Capital.»En Talja Blokland y Mike Savage (eds.), Networked Urbanism. Social Capital in the City. Hampshire: Ashgate.

Inkeles, Alex. 2000. «Measuring Social Capital and Its Consequences.» Policy Sciences, 33 (3-4): 245-268.

Jordana, Jacint. 2000. «Instituciones y Capital Social ¿qué explica qué?» Revista Española de Ciencia Política, 1 (2): 187-210.

Krishna, Anirudh. 2002. «Enhancing Political Participation in Democracies: What is the Role of Social Capital?», Comparative Political Studies, 35: 437-460.

Lake, Ronald La Due y Robert Huckfeldt. 1998. «Social Capital, Social Networks, and Political Participation", Political Psychology. 19 (3): 567-584.

Levi, Margaret. 1996. «Social and Unsocial Capital. A review essay of Robert Putnam's Making Democracy Work», Politics and Society, 24 (1): 45-55.

Lin, Nan. 2000. "Inequality in Social Capital», Contemporary Sociology, 29 (6): $785-795$.

Lin, Nan. 2001. Social Capital. A Theory of Social Structure and Action. Cambridge: Cambridge University Press.

Lowndes, Vivien. 2003. "Getting on or getting by? Women, social capital and political participation». Paper for presentation to Gender and Social Capital Conference. Winnipeg, Canada: University of Manitoba.

Lowndes, Vivien y Lawrence Pratchett. 2009. «Factores que explican la participación: comparando recursos, relaciones y normas en cinco países europeos», en Marc Parés (coord.), Participación y calidad democrática. Evaluando las nuevas formas de democracia participativa. Barcelona: Ariel Ciencia Política.

Lowndes, Vivien, Lawrence Pratchett y Gerry Stoker. 2006. «Diagnosing and Remedying the Failings of Official Participation Schemes: The CLEAR Framework", Social Policy and Society, 5: 281-91.

Martínez, Miguel Ángel y Elísabeth Lorenzi. 2012. "Autonomous activist-research. The case of the squatters' movement in Madrid", Revista Internacional de Sociologia (RIS). Special issue on methodological challenges in participation research, 70 (extra 2): 165-184.

Molenaers, Nadia. 2005. "Tracing the Contradictions. Associational Life versus Informal Networks in a Third World Context», en Sigrid Roßteutscher (ed.), Democracy 
and the Role of Associations. Political, Organizational and Social Contexts. Londres y Nueva York: Routledge/ECPR Studies in European Political Science.

Morales Díez, Laura. 2004. "El asociacionismo político en Europa», Revista Zona Abierta, 106-107: 7-63.

Mota, Fabiola y Joan Subirats. 2000. «El quinto elemento: el capital social de las comunidades autónomas. Su impacto sobre el funcionamiento del sistema político autonómico», Revista Española de Ciencia Política, 1 (2): 123-158.

Narayan, Deepa y Mark Woolcock. 2000. «Social Capital: Implications for Development Theory, Research, and Policy», The World Bank Research Observer, 15 (2): 225-249.

Norris, Pippa, y Ronald Inglehart. 2006. «Gendering Social Capital: Bowling in Women’s Leagues?», en B. O’Neill y E. Gidengil, Gender and Social Capital. Nueva York: Routledge.

Ostrom, Elinor. 1990. Governing the Commons: The Evolution of Institutions for Collective Action. Cambridge: Cambridge University Press.

Ostrom, Elinor. 2000. «Social Capital: A fad or a fundamental concept?», en Partha Dasgupta y Ismail Serageldin, (eds.), Social Capital: A Multifaceted Perspective. Washington DC: World Bank.

Ostrom, Elinor y T. K. Ahn. 2003. «Una perspectiva del capital social desde las ciencias sociales capital social y acción colectiva», Revista Mexicana de Sociología, 65: $155-233$.

Portes, Alejandro. 1998. «Social Capital: Its Origins and Applications in Modern Sociology", Annual Review of Sociology, 24: 1-24.

Putnam, Robert. 1993. Making Democracy Work. Civic Traditions in Modern Italy. Princeton: Princeton University Press.

Putnam, Robert. 2000. Bowling alone. The collapse and revival of American community. Nueva York: Simon and Schuster.

Roßteutscher, Sigrid. 2008. «Social Capital and Civic Engagement: A Comparative Perspective», en Dario Castiglione, Jan W. Van Deth y Guglielmo Wolleb (eds.), The Handbook of Social Capital. Oxford: Oxford University Press.

Savage, Mike, Gaynor Bagnall y Brian Longhurst. 2005. Globalisation and Belonging. Londres: Sage.

Sengupta, Urmi y Sujeet Sharma. 2012. «Urban development and social capital: lessons from Kathmandu», en Joseph D. Lewandowski y Gregory W. Streich (eds.), Urban social capital: civil society and city life. Londres: Routledge.

Van Deth, Jan W. 2003. «Measuring social capital: Orthodoxies and continuing controversies», International Journal of Social Research Methodology, 6 (1): 79-92.

Van Deth, Jan W. 2008. "Measuring social capital», en Dario Castiglione, Jan W. Van Deth y Guglielmo Wolleb (eds.), The Handbook of Social Capital. Oxford: Oxford University Press.

Vásquez González, Y. Lorena. 2013. Participación ciudadana y democracia local. La influencia del contexto politico y social. Estudio comparado Bogotá y Madrid [tesis doctoral]. Fabiola Consejero (dir.), Universidad de Alcalá, Alcalá de Henares. 
Velásquez, Fabio y Esperanza González. 2004. Encuentros con el futuro. Cuarto ejercicio de planeación participativa en Bogotá. Bogotá: Fundación Corona y Foro Nacional por Colombia.

Verba, Sidney, Kay Lehman Schlozman, y Henry Brady. 1995. Voice and Equality: Civic Voluntarism in American Politics. Cambridge, Mass.: Harvard University Press.

Walliser, Andrés. 2003. Participación y Ciudad. Madrid: Instituto Juan March de Estudios e Investigaciones. Centro de Estudios Avanzados en Ciencias Sociales.

Walliser, Andrés. 2013. «New urban activisms in Spain: reclaiming public space in the face of crises», Policy and Politics, 43 (3): 329-350.

Wampler, Brian y Leonardo Avritzer. 2004. «Participatory publics: Civil society and new institutions in democratic Brazil», Comparative Politics, 36 (3): 291-312.

Woolcock, Mark. 1998. «Social Capital and Economic Development: Towards a Theoretical Synthesis and a Policy Framework», Theory and Society, 27 (2): 151-208.

Woolcock, Mark. 2010. "The rise and routinization of social capital, 1988-2008", Annual Review of Political Science, 13: 469-487.

Woolcock, Michael y Veronica Nyhan Jones. 2009. «Mixed methods assessment», en Gert Tingaard Svendsen y Gunnar Lind Haase Svendsen (eds.), Handbook of Social Capital. The Troika of Sociology, Political Science and Economics. Northampton, MA: Edward Elgar Publishing.

Presentado para evaluación: 11 de enero de 2018.

Aceptado para publicación: 25 de octubre de 2018.

\section{Y. LORENA VÁSQUEZ-GONZÁLEZ}

lorena.vasquezg@utadeo.edu.co

Profesora titular del Departamento de Ciencias Políticas y Relaciones Internacionales de la Universidad de Bogotá Jorge Tadeo Lozano. Investigadora asociada del Instituto de Estudios Latinoamericanos, Universidad de Alcalá. Doctora en Estudios Latinoamericanos por la Universidad de Alcalá. Licenciada en Ciencia Política por la Universidad Nacional de Colombia y máster en Administración y Gerencia Pública por el Instituto Nacional de Administración Pública de España (INAP) y por la Universidad de Alcalá. Diploma de Estudios Avanzados, Doctorado en Teoría Política, Democrática y Administración Pública de la Universidad Autónoma de Madrid. Investigadora visitante en la Universidad de Bristol. Sus líneas de investigación son: participación ciudadana directa en gobiernos locales, gobernanza urbana, acción colectiva, capital social, ciudadanía y política comparada. 